\title{
An efficient solid phase one port synthesis of Novel triazolo[1,5-a]pyrimidine derivatives from 4-(4- aminophenyl)morpholin-3-one and evaluation of their antimicrobial activity
}

\author{
Devang R. Prajapati, Kalpesh V. Vilapara, Yogesh T. Naliapara* \\ Department of Chemistry, Saurashtra University, Rajkot, Gujarat, India \\ *E-mail addres: naliaparachem@yahoo.com
}

\begin{abstract}
A series of novel triazolo[1,5-a]pyrimidine derivatives was synthesized from 5-amino-1,2,4triazole and biologically active morpholinone amine in excellent yield as promising class of antimicrobial agents. The antimicrobial activities were investigated against Escherichia coli, Staphylococcus aureus, Pseudomonas aeruginosa, Staphylococcus pyogen, Candida albicans, Aspergillusniger, Aspergillusclavatus and compared with standard drugs Ampicillin, Chloramphenicol, Norfloxacin and Griseofulvin. All the synthesized compounds were characterized by IR, ${ }^{1} \mathrm{H}$ NMR, ${ }^{13} \mathrm{C}$ and mass spectroscopy. The result of antimicrobial activity data revealed that compound 4f,4g and $4 \mathrm{i}$ were found more active against bacterial species and compound 4c, 4d, 4g, 4i and 4 jwere found more active against fungal strain, while other compounds shows moderate to law activity against microbes.
\end{abstract}

Keywords: 1,2,4-triazole; 4-(4-aminophenyl)morpholin-3-one; antibacterial; antifungal; biological assay

\section{INTRODUCTION}

Various fused pyrimidines were studied in the past decade and were found to possess remarkable pharmacological properties like purines, pteridines, quinazolines, triazolopyrimidines, pyrazolopyrimidines, pyrimidoazepines, furopyrimidines, pyridopyrimidines and pyrrolopyrimidines.

The condensation of a pyrimidine ring with another ring of 1,2,4-triazole gives rise to the formation of bicyclic heterocycles known as 1,2,4-triazolopyrimidines. 1,2,4-triazolo[1,5a]pyrimidine derivatives are thermodynamically more stable and, thus, the most studied ones [1], a few of them being commercially available.

Revisions surveying the synthesis, reactivity, spectroscopic characterization and crystallographic studies of 1,2,4-triazolo[1,5-c]pyrimidines [2], 1,2,4-triazolo[4,3a]pyrimidines [3] and 1,2,4-triazolo[4,3-c]pyrimidines [4] have also been published.

Recently, 1,2,4-triazolo[1,5-a]pyrimidines have aroused increasing attention from the chemical and biological points of view due to their diverse pharmacological activities such as 
anti-inflammatory [5], antimalarial [6], antifungal effect [7], macrophage activation [8], antitumor potency [9-12], antimicrobial [13-16,20-24] and inhibition of KDR kinase [17]. They have proven to be promising anticancer [18] agents with dual mechanisms of tubulin polymerization promotion [9-10] as well as cyclin dependent kinases 2 inhibition [19].

\section{RESULTS AND DISCUSSION}

\section{Chemistry:}

4-methyl-3-oxo-N-(4-(3-oxomorpholino)phenyl)pentanamide (2a) were prepared by reacting 4-(4-aminophenyl)morpholin-3-one (1a) and methyl-4-methyl-3-oxopentanoate in toluene with a catalytic amount of $\mathrm{NaOH} / \mathrm{KOH}$ (Scheme 1). The reaction mixture was refluxed for $15-20 \mathrm{~h}$.<smiles>Nc1ccc(N2CCOCC2=O)cc1</smiles>

$1 \mathrm{a}$<smiles>COC(=O)CC(=O)C(C)C</smiles>

$\underset{\begin{array}{l}\text { Toluene/KOH } \\ \text { refulx }\end{array}}{\longrightarrow}$

Scheme 1. Synthesis of 4-methyl-3-oxo-N-(4-(3-oxomorpholino)phenyl)pentanamide.<smiles>[R]c1cccc(C=O)c1</smiles>

Scheme 2. Synthesis of 5-isopropyl-N-(4-(3-oxomorpholino)phenyl)-7-phenyl-4,7-dihydro$[1,2,4]$ triazolo[1,5-a]pyrimidine-6-carboxamideby using 4H-1,2,4-triazol-3-amine.

Reaction of acetoacetamide (2a) derivative with various aromatic aldehydes (3a-t) and 1,2,4-triazol-amine in presence of trace amount of dimethyl formamide at high temperature(fusion) gives the novel triazolo[1,5-a]pyrimidinederivatives $(4 a-t)$ with excellent yields (Scheme 2). All the synthesized compounds were characterized by various spectroscopic techniques like ${ }^{1} \mathrm{H}$ NMR, ${ }^{13} \mathrm{C}$ NMR, IR and Mass. 


\section{Biology:}

All the synthesized compounds were tested against different bacterial and fungal strains i.e. Pseudomonas aeruginosa, Proteus vulgaris, Escherichia Coli, Staphylococcus aureus, Candida albicanfor their in vitro antibacterial activity.Well Diffusion/Agar Cup Method was usedand results are listed in Table 2. The investigation of antibacterial and antifungal screening data revealed that all the tested compounds $4 \mathrm{a}-\mathrm{t}$ showed moderate to potent activity. The compounds $4 \mathrm{f}, 4 \mathrm{~g}$ and $4 \mathrm{i}$ showedcomparatively good activity against all the bacterial strains.

\section{1. Experimental}

Thin-layer chromatography was accomplished on $0.2-\mathrm{mm}$ precoated plates of silica gel G60 $\mathrm{F}_{254}$ (Merck). Visualization was made with UV light $(254$ and $365 \mathrm{~nm}$ ) or with an iodine vapor. IR spectra were recorded on a FTIR-8400 spectrophotometer using DRS prob. ${ }^{1} \mathrm{H}(400$ $\mathrm{MHz})$ and ${ }^{13} \mathrm{C}(400 \mathrm{MHz}) \mathrm{NMR}$ spectra were recorded on a Bruker AVANCE II spectrometer in DMSO. Chemical shifts are expressed in $\delta$ ppm downfield from TMS as an internal standard. Mass spectra were determined using direct inlet probe on a GCMS-QP 2010 mass spectrometer (Shimadzu). Solvents were evaporated with a BUCHI rotary evaporator. Melting points were measured in open capillaries and are uncorrected.

\section{1. 1. General procedure for the synthesis of 4-methyl-3-oxo-N-(4-(3-oxomorpholino) phenyl)pentanamide (2a)}

A mixture of 4-(4-aminophenyl)morpholin-3-one (10 mmol),methyl 4-methyl-3oxopentanoate $(10 \mathrm{mmol})$ and catalytic amount of sodium or potassium hydroxide lie $(10 \%)$ in toluene $(50 \mathrm{ml})$ was refluxed at $110{ }^{\circ} \mathrm{C}$ for $12-15 \mathrm{~h}$. The reaction was monitored by TLC. After completion of reaction, the solid was precipitated out,filtered it washed with n-hexane giving $2 \mathrm{a}$.

\section{1. 2. General procedure for the synthesis of substituted triazolopyrimidines (4a-t)}

A mixture of the aminoazole (0.01 mol), 4-methyl-3-oxo-N-(4-(3-oxomorpholino) phenyl)pentanamide $(0.01 \mathrm{~mol}) \mathbf{2 a}$ and an appropriate aromatic aldehyde $(0.01 \mathrm{~mol})$ 3a-t was fused in presence of trace amount of DMF for 12-15 min. After cooling, methanol $(\sim 10 \mathrm{~mL})$ was added. The reaction mixture was allowed to stand overnight and then filtered to give the solid triazolopyrimidine products 4a-t, which were crystallized from ethanol and subsequently dried in air.

\section{1. 3. Spectroscopic data for the compounds (4a-t):}

5-isopropyl-N-(4-(3-oxomorpholino)phenyl)-7-phenyl-4,7-dihydro-[1,2,4]triazolo[1,5a]pyrimidine-6-carboxamide (4a): White solid; $\mathrm{mp}$ 240-242 ${ }^{\circ} \mathrm{C}$; $\mathrm{R}_{\mathrm{f}} 0.53$ (4:6 hexaneEtOAc); IR (KBr): 3643, 3375, 3061, 2922, 2858, 1919, 1790, 1635, 1552, 1504, 1458, 1294, 1192, 1076, 1026, 997, 856,785, 696, $580 \mathrm{~cm}^{-1}$; ${ }^{1} \mathrm{H}$ NMR: $\delta 1.185-1.307\left(\mathrm{~d}, 6 \mathrm{H},\left(\mathrm{CH}_{3}\right)_{2}, j=\right.$ $6.88 \mathrm{~Hz}), 3.306-3.396(\mathrm{~m}, 1 \mathrm{H},-\mathrm{CH}), 3.652-3.678\left(\mathrm{t}, 2 \mathrm{H},-\mathrm{CH}_{2}\right.$ - in morpholinone ring), 3.937-3.962 (t, $2 \mathrm{H},-\mathrm{CH}_{2}$ - in morpholinone ring), $4.174\left(\mathrm{~s}, 2 \mathrm{H},-\mathrm{CH}_{2}\right.$ - in morpholinone ring near ketone), $6.528(\mathrm{~s}, 1 \mathrm{H},-\mathrm{CH}$ pyrimidine ring), 7.205-7.222 (t, 2H, Ar-H, $=4.92 \mathrm{~Hz})$, 7.239-7.255 (d, 2H, Ar-H, j= 5.0 Hz), 7.254-7.281 (t, 1H, Ar-H), 7.296-7.316 (d, 2H, Ar-H ring nearby -NHCO-), 7.520-7.542 (d, 2H, Ar-H ring nearby -NHCO-), $7.593(\mathrm{~s}, 1 \mathrm{H},-\mathrm{CH}$ triazole ring), 9.926 (s, 1H, -NH pyrimidine ring), 10.002 (s, 1H, -NH amide); ${ }^{13} \mathrm{C}$ NMR (400 
MHz, DMSO): 19.54, 28.75, 38.88, 39.09, 39.29, 39.50, 39.71, 39.92, 40.13, 49.09, 60.69, $63.47,67.73,102.53,119.83,125.62,126.94,127.97,128.37,136.65,137.32,140.45$, 143.99, 148.32, 149.71, 165.83; MS (m/z): $458\left(\mathrm{M}^{+}\right)$; Anal. Calcd for $\mathrm{C}_{25} \mathrm{H}_{26} \mathrm{~N}_{6} \mathrm{O}_{3}: \mathrm{C}, 65.49$; H, 5.72; N, 18.33; Found: C, 65.30; H, 5.70; N, 18.10 .

7-(4-chlorophenyl)-5-isopropyl-N-(4-(3-oxomorpholino)phenyl)-4,7-dihydro[1,2,4]

triazolo[1,5-a]pyrimidine-6-carboxamide (4b): White solid; $\mathrm{mp}$ 245-248 ${ }^{\circ} \mathrm{C}$; $\mathrm{R}_{\mathrm{f}} 0.48$ (4:6 hexane-EtOAc); IR (KBr): 3742, 3377, 3095, 2974, 2861,2155, 1919, 1735, 1550, 1461, 1236, 1192, 1001, 923, 833, 783, 636, $588 \mathrm{~cm}^{-1}$; MS $(\mathrm{m} / \mathrm{z}): 492\left(\mathrm{M}^{+}\right)$; Anal. Calcd for $\mathrm{C}_{25} \mathrm{H}_{25} \mathrm{ClN}_{6} \mathrm{O}_{3}: \mathrm{C}, 60.91 ; \mathrm{H}, 5.11 ; \mathrm{N}, 17.05$; Found: C, 60.85; H, 5.10; N, 17.10 .

\section{7-(4-fluorophenyl)-5-isopropyl-N-(4-(3-oxomorpholino)phenyl)-4,7-dihydro[1,2,4]}

triazolo[1,5-a]pyrimidine-6-carboxamide (4c): White solid; mp260-265 ${ }^{\circ} \mathrm{C} ; \mathrm{R}_{\mathrm{f}} 0.52$ (4:6 hexane-EtOAc); IR (KBr): 3738, 3614, 3383, 3048, 2933, 2864, 1919, 1730, 1651, 1557 , 1520, 1454, 1291, 1177, 1072, 999, 895, 792, 646, $559 \mathrm{~cm}^{-1}$; MS $(\mathrm{m} / z): 476\left(\mathrm{M}^{+}\right)$; Anal. Calcd for $\mathrm{C}_{25} \mathrm{H}_{25} \mathrm{ClN}_{6} \mathrm{O}_{3}$ : C, 63.01; H, 5.29; N, 17.64; Found: C, 63.05; H, 5.35; N, 17.80 .

\section{7-(2-chlorophenyl)-5-isopropyl-N-(4-(3-oxomorpholino)phenyl)-4,7-dihydro[1,2,4]}

triazolo[1,5-a]pyrimidine-6-carboxamide (4d): White solid; mp 235-237 ${ }^{\circ} \mathrm{C} ; \mathrm{R}_{\mathrm{f}} 0.50(4: 6$ hexane-EtOAc); IR (KBr): 3631, 3248, 3107, 3052, 2964, 2868, 1784, 1649, 1579, 1533, $1475,1247,1193,1076,842,767,657,559 \mathrm{~cm}^{-1}$; ${ }^{1} \mathrm{H}$ NMR: $\delta 1.193-1.312\left(\mathrm{~d}, 6 \mathrm{H},\left(\mathrm{CH}_{3}\right)_{2}, j=\right.$ $6.92 \mathrm{~Hz}), 3.274-3.408\left(\mathrm{~m}, 1 \mathrm{H},-\mathrm{CH}\right.$ of isopropyl gp.), 3.666-3.691 (t, $2 \mathrm{H},-\mathrm{CH}_{2^{-}}$in morpholinone ring), 3.948-3.973 (t, $2 \mathrm{H},-\mathrm{CH}_{2}$ - in morpholinone ring), $4.179\left(\mathrm{~s}, 2 \mathrm{H},-\mathrm{CH}_{2}\right.$ - in morpholinone ring near ketone), $6.528(\mathrm{~s}, 1 \mathrm{H},-\mathrm{CH}$ pyrimidine ring), 7.187-7.283 (m, 4H, Ar$\mathrm{H}, j=4.96 \mathrm{~Hz}), 7.295-7.313(\mathrm{~d}, 2 \mathrm{H}$, Ar-H ring nearby $-\mathrm{NHCO}-, j=1.52 \mathrm{~Hz}), 7.332-7.539(\mathrm{~d}$, $2 \mathrm{H}$, Ar-H ring nearby $-\mathrm{NHCO}-, j=7.96 \mathrm{~Hz}), 8.213(\mathrm{~s}, 1 \mathrm{H},-\mathrm{CH}$ triazole ring), $10.004(\mathrm{~s}, 1 \mathrm{H}$, -NH pyrimidine ring), 10.031 (s, 1H, -NH amide); ${ }^{13} \mathrm{C}$ NMR (400 MHz, DMSO): 19.52, 19.76, 28.91, 38.87, 39.08, 39.28, 39.49, 39.70, 39.91, 40.12, 49.08, 60.11, 63.47, 67.73, 78.44, 78.77, 79.10, 101.88, 119.93, 125.63, 126.88, 128.10, 130.21, 133.27, 136.78, 137.14, 142.53, 144.27, 148.28, 149.95, 164.87, 165.83; MS $(\mathrm{m} / \mathrm{z}): 492\left(\mathrm{M}^{+}\right)$; Anal. Calcd for $\mathrm{C}_{25} \mathrm{H}_{25} \mathrm{ClN}_{6} \mathrm{O}_{3}: \mathrm{C}, 60.91 ; \mathrm{H}, 5.11 ; \mathrm{N}, 17.05$; Found: C, 59.76; H, 5.20; N, 17.10 .

\section{7-(2,5-dimethoxyphenyl)-5-isopropyl-N-(4-(3-oxomorpholino)phenyl)-4,7-dihydro-}

$[1,2,4]$ triazolo[1,5-a]pyrimidine-6-carboxamide(4e): White solid; mp $238-240{ }^{\circ} \mathrm{C} ; \mathrm{R}_{\mathrm{f}} 0.43$ (4:6 hexane-EtOAc); IR (KBr): 3642, 3239, 3095, 2962, 2831, 1789, 1668, 1581, 1543, 1467, 1276, 1184, 1047, 1027, 837, 709, 679, $547 \mathrm{~cm}^{-1}$; MS $(\mathrm{m} / \mathrm{z}): 518\left(\mathrm{M}^{+}\right)$; Anal. Calcd for $\mathrm{C}_{27} \mathrm{H}_{30} \mathrm{~N}_{6} \mathrm{O}_{5}$ : C, 62.54; H, 5.83; N, 16.21; Found: C, 62.00; H, 5.75; N, 16.30.

5-isopropyl-N-(4-(3-oxomorpholino)phenyl)-7-(p-tolyl)-4,7-dihydro-[1,2,4]triazolo[1,5a]pyrimidine-6-carboxamide (4f): White solid; $\mathrm{mp} 240-245{ }^{\circ} \mathrm{C}$; $\mathrm{R}_{\mathrm{f}} 0.51$ (4:6 hexaneEtOAc); IR (KBr): 3379, 3284, 3099, 2978, 2870, 1658, 1610, 1597, 1408, 1348, 1238, 1192 , 1072, 786, 684, 648, 588, $511 \mathrm{~cm}^{-1}$; ${ }^{1} \mathrm{H}$ NMR: $\delta 1.177-1.300\left(\mathrm{~d}, 6 \mathrm{H},\left(\mathrm{CH}_{3}\right)_{2}, j=7.04 \mathrm{~Hz}\right)$, $2.252\left(\mathrm{~s}, 3 \mathrm{H},-\mathrm{CH}_{3}\right.$ gp. $), 3.301-3.388(\mathrm{~m}, 1 \mathrm{H},-\mathrm{CH}), 3.659-3.685\left(\mathrm{t}, 2 \mathrm{H},-\mathrm{CH}_{2-}\right.$ in morpholinone ring), 3.946-3.971 (t, $2 \mathrm{H},-\mathrm{CH}_{2}$ - in morpholinone ring), $4.174\left(\mathrm{~s}, 2 \mathrm{H},-\mathrm{CH}_{2}\right.$ - in morpholinone ring near ketone), $6.482(\mathrm{~s}, 1 \mathrm{H},-\mathrm{CH}$ pyrimidine ring), 7.095-7.117 (d, 2H, Ar$\mathrm{H}, j=8.68 \mathrm{~Hz}), 7.211-7.218(\mathrm{~d}, 2 \mathrm{H}, \mathrm{Ar}-\mathrm{H}, j=2.6 \mathrm{~Hz}), 7.240-7.527(\mathrm{~d}, 2 \mathrm{H}, \mathrm{Ar}-\mathrm{H}$ ring nearby -NHCO-), 7.549-7.573 (d, 2H, Ar-H ring nearby -NHCO-), 8.213 (s, 1H, -CH triazole ring), 9.875 (s, 1H, -NH pyrimidine ring), 9.983 (s, 1H, - NH amide) ${ }^{13} \mathrm{C}$ NMR (400 MHz, 
DMSO):19.55, 19.78, 20.66, 28.70, 38.88, 39.09, 39.30, 39.51, 39.72, 39.92, 40.13, 49.10, $60.40,63.47,67.72,78.45,78.78,79.11,102.61,119.77,125.61,126.87,128.94,136.62$, 137.30, 137.36, 137.58, 143.89, 148.23, 149.62, 165.82; MS $(\mathrm{m} / \mathrm{z}): 472\left(\mathrm{M}^{+}\right)$; Anal. Calcd for $\mathrm{C}_{26} \mathrm{H}_{28} \mathrm{~N}_{6} \mathrm{O}_{3}$ : C, 66.09; H, 5.97; N, 17.78; Found: C, 66.10; H, 5.91; N, 17.90.

7-(3,4-dimethoxyphenyl)-5-isopropyl-N-(4-(3-oxomorpholino)phenyl)-4,7-dihydro$[1,2,4]$ triazolo[1,5-a]pyrimidine-6-carboxamide (4g): White solid; mp 240-243 ${ }^{\circ} \mathrm{C} ; \mathrm{R}_{\mathrm{f}} 0.42$ (4:6 hexane-EtOAc); IR (KBr): 3642, 3232, 3071, 2968, 2876, 1784, 1649, 1591, 1521, 1411, 1261, 1128, 1028, 875, 732, 659, $555 \mathrm{~cm}^{-1}$; MS $(\mathrm{m} / z): 518\left(\mathrm{M}^{+}\right)$; Anal. Calcd for $\mathrm{C}_{27} \mathrm{H}_{30} \mathrm{~N}_{6} \mathrm{O}_{5}$ : C, 62.54; H, 5.83; N, 16.21; Found: C, 62.70; H, 5.90; N, 16.17.

5-isopropyl-7-(4-methoxyphenyl)-N-(4-(3-oxomorpholino)phenyl)-4,7-dihydro-[1,2,4] triazolo[1,5-a]pyrimidine-6-carboxamide (4h): White solid; $\mathrm{mp} 225-228{ }^{\circ} \mathrm{C} ; \mathrm{R}_{\mathrm{f}} 0.43$ (4:6 hexane-EtOAc); IR (KBr): 3610, 3244, 3113, 3055, 2933, 2868, 1791, 1622, 1593, 1321, 1271, 1205, 1166, 1028, 827, 794, 659, $565 \mathrm{~cm}^{-1}$; MS $(\mathrm{m} / \mathrm{z}): 488\left(\mathrm{M}^{+}\right)$; Anal. Calcd for $\mathrm{C}_{26} \mathrm{H}_{28} \mathrm{~N}_{6} \mathrm{O}_{4}$ : C, 63.92; H, 5.78; N, 17.20; Found: C, 64.00; H, 5.60; N, 17.10.

5-isopropyl-7-(2-nitrophenyl)-N-(4-(3-oxomorpholino)phenyl)-4,7-dihydro-[1,2,4]

triazolo[1,5-a]pyrimidine-6-carboxamide (4i): White solid; mp $248-250{ }^{\circ} \mathrm{C}$; $\mathrm{R}_{\mathrm{f}} 0.48$ (4:6 hexane-EtOAc); IR (KBr): 3634, 3527, 3228, 3099, 2958, 2872, 1778, 1664, 1583, 1531, 1473, 1356, 1317, 1253, 1199, 1072, 1020, 995, 844, 731, 684, 547 $\mathrm{cm}^{-1}$; MS $(\mathrm{m} / \mathrm{z}): 503\left(\mathrm{M}^{+}\right)$; Anal. Calcd for $\mathrm{C}_{25} \mathrm{H}_{25} \mathrm{~N}_{7} \mathrm{O}_{5}$ : C, 59.63; H, 5.00; N, 19.47; Found: C, 59.50; H, 5.10; N, 19.55 .

7-(2,4-dichlorophenyl)-5-isopropyl-N-(4-(3-oxomorpholino)phenyl)-4,7-dihydro-

$[\mathbf{1 , 2 , 4}]$ triazolo[1,5-a]pyrimidine-6-carboxamide (4j): White solid; $\mathrm{mp} 250-255^{\circ} \mathrm{C} ; \mathrm{R}_{\mathrm{f}} 0.40$ (4:6 hexane-EtOAc); IR (KBr): 3610, 3236, 3080, 2933, 2891, 1763, 1630, 1570, 1538, 1470, 1276, 1170, 1072, 1041, 800, 870, 736, 667, 589 $\mathrm{cm}^{-1}$; MS $(\mathrm{m} / \mathrm{z}): 527\left(\mathrm{M}^{+}\right)$; Anal. Calcd for $\mathrm{C}_{25} \mathrm{H}_{24} \mathrm{Cl}_{2} \mathrm{~N}_{6} \mathrm{O}_{3}$ : C, 56.93; H, 4.59; N, 15.93; Found: C, 57.00; H, 4.50; N, 16.01 .

7-(3,4-dimethylphenyl)-5-isopropyl-N-(4-(3-oxomorpholino)phenyl)-4,7-dihydro$[1,2,4]$ triazolo[1,5-a]pyrimidine-6-carboxamide (4k): Whitesolid; mp 252-255 ${ }^{\circ} \mathrm{C} ; \mathrm{R}_{\mathrm{f}} 0.44$ (4:6 hexane-EtOAc); IR (KBr): 3630, 3240, 3090, 2936, 2890, 1763, 1640, 1580, 1542, 1480, 1276, 1170, 1074, 1043, 800, 880, 735, 662, $590 \mathrm{~cm}^{-1}$; MS $(\mathrm{m} / \mathrm{z}): 527\left(\mathrm{M}^{+}\right)$; Anal. Calcd for $\mathrm{C}_{25} \mathrm{H}_{24} \mathrm{Cl}_{2} \mathrm{~N}_{6} \mathrm{O}_{3}$ : C, 56.93; H, 4.59; N, 15.93; Found: C, 57.01; H, 4.50; N, 15.90 .

7-(2-chlorophenyl)-5-isopropyl-N-(4-(3-oxomorpholino)phenyl)-4,7-dihydro-[1,2,4] triazolo[1,5-a]pyrimidine-6-carboxamide (4l): White solid; $\mathrm{mp} 236-237{ }^{\circ} \mathrm{C} ; \mathrm{R}_{\mathrm{f}} 0.43$ (4:6 hexane-EtOAc); IR (KBr): 3233, 3188, 3064, 2918, 2864, 1741, 1680, 1624, 1542, 1449, 1390, 1280, 1213, 1136, 1035, 966, 835, 768, 670, $595 \mathrm{~cm}^{-1}$; MS $(\mathrm{m} / \mathrm{z}): 492\left(\mathrm{M}^{+}\right)$; Anal. Calcd for $\mathrm{C}_{25} \mathrm{H}_{25} \mathrm{ClN}_{6} \mathrm{O}_{3}$ : C, 60.91; H, 5.11; N, 17.05; Found: C, 60.85; H, 5.20; N, 17.10.

5-isopropyl-N-(4-(3-oxomorpholino)phenyl)-7-(m-tolyl)-4,7-dihydro-[1,2,4]triazolo[1,5a]pyrimidine-6-carboxamide (4m): White solid; mp2 42-245 ${ }^{\circ} \mathrm{C} ; \mathrm{R}_{\mathrm{f}} 0.49$ (4:6 hexaneEtOAc); IR (KBr): 3290, 3045, 2936, 1730, 1633, 1555, 1512, 1465, 1370, 1290, 1195, 990, 815, 769, 669, $515 \mathrm{~cm}^{-1}$; MS $(\mathrm{m} / z): 472\left(\mathrm{M}^{+}\right)$; Anal. Calcd for $\mathrm{C}_{26} \mathrm{H}_{28} \mathrm{~N}_{6} \mathrm{O}_{3}$ : C, 66.09; H, 5.97; N, 17.78; Found: C, 66.15; H, 5.93; N, 17.80. 
7-(2,3-dimethylphenyl)-5-isopropyl-N-(4-(3-oxomorpholino)phenyl)-4,7-dihydro-[1,2,4] triazolo[1,5-a]pyrimidine-6-carboxamide (4n): White solid; mp 255-258 ${ }^{\circ} \mathrm{C} ; \mathrm{R}_{\mathrm{f}} 0.48$ (4:6 hexane-EtOAc); IR (KBr): 3242, 3180, 3054, 2928, 2857, 1740, 1675, 1632, 1437, 1391, 1270, 1225, 1144, 1048, 951, 843, 779, 683, 591 $\mathrm{cm}^{-1}$; MS $(\mathrm{m} / \mathrm{z}): 487\left(\mathrm{M}^{+}\right)$; Anal. Calcd for $\mathrm{C}_{27} \mathrm{H}_{30} \mathrm{~N}_{6} \mathrm{O}_{3}$ : C, 66.65; H, 6.21; N, 17.27; Found: C, 66.67; H, 6.31; N, 17.33.

7-(2-fluorophenyl)-5-isopropyl-N-(4-(3-oxomorpholino)phenyl)-4,7-dihydro-

$[1,2,4]$ triazolo[1,5-a]pyrimidine-6-carboxamide (4o): White solid; $\mathrm{mp} 262-265{ }^{\circ} \mathrm{C} ; \mathrm{R}_{\mathrm{f}} 0.47$ (4:6 hexane-EtOAc); IR (KBr): 3277, 3155, 3058, 2920, 2874, 1670, 1590, 1478, 1327, 1288 , 1210, 1192, 1022, 976, 817, 747, 662, 556 $\mathrm{cm}^{-1}$; MS $(\mathrm{m} / \mathrm{z}): 476\left(\mathrm{M}^{+}\right)$; Anal. Calcd for $\mathrm{C}_{25} \mathrm{H}_{25} \mathrm{FN}_{6} \mathrm{O}_{3}$ : C, 63.01; H, 5.29; N, 17.64; Found: C, 63.11; H, 5.35; N, 17.70.

5-isopropyl-7-(2-nitrophenyl)-N-(4-(3-oxomorpholino)phenyl)-4,7-dihydro-[1,2,4] triazolo[1,5-a]pyrimidine-6-carboxamide (4p): Off White solid; mp 250-252 ${ }^{\circ} \mathrm{C} ; \mathrm{R}_{\mathrm{f}} 0.40$ (4:6 hexane-EtOAc); IR (KBr): 3290, 3150, 3063, 2929, 2870, 1671, 1550, 1482, 1370, 1278, 1186, 1076, 965, 831, 750, 675, 525 $\mathrm{cm}^{-1}$; MS (m/z): $503\left(\mathrm{M}^{+}\right)$; Anal. Calcd for $\mathrm{C}_{25} \mathrm{H}_{25} \mathrm{~N}_{7} \mathrm{O}_{5}$ : C, 59.63; H, 5.00; N, 19.47; Found: C, 59.50; H, 4.92; N, 19.60 .

7-(3-chloro-4-fluorophenyl)-5-isopropyl-N-(4-(3-oxomorpholino)phenyl)-4,7-dihydro$[1,2,4]$ triazolo[1,5-a]pyrimidine-6-carboxamide (4q): White solid; $\mathrm{mp} 264-266{ }^{\circ} \mathrm{C} ; \mathrm{R}_{\mathrm{f}} 0.45$ (4:6 hexane-EtOAc); IR (KBr): 3240, 3190, 3068, 2950, 2860, 1690, 1597, 1590, 1490, 1280, 1185, 1085, 1050, 873, 789, 680, $596 \mathrm{~cm}^{-1}$; MS $(\mathrm{m} / \mathrm{z}): 510\left(\mathrm{M}^{+}\right)$; Anal. Calcd for $\mathrm{C}_{25} \mathrm{H}_{24} \mathrm{ClFN}_{6} \mathrm{O}_{3}$ : C, 58.77; H, 4.73; N, 16.45; Found: C, 58.82; H, 4.69; N, 16.50 .

7-(4-chloro-2-nitrophenyl)-5-isopropyl-N-(4-(3-oxomorpholino)phenyl)-4,7-dihydro$[1,2,4]$ triazolo[1,5-a]pyrimidine-6-carboxamide (4r): Off white solid; mp $260-265{ }^{\circ} \mathrm{C}$; $\mathrm{R}_{\mathrm{f}}$ 0.39 (4:6 hexane-EtOAc); IR (KBr): 3250, 3189, 3072, 2939, 2879, 1730, 1667, 1550, 1470, 1350, 1310, 1290, 1160, 1073, 884, 778, 677, $592 \mathrm{~cm}^{1}$; MS $(\mathrm{m} / \mathrm{z}): 537\left(\mathrm{M}^{+}\right)$; Anal. Calcd for $\mathrm{C}_{25} \mathrm{H}_{24} \mathrm{ClN}_{7} \mathrm{O}_{5}$ : C, 55.82; H, 4.50; N, 18.23; Found: C, 55.90; H, 4.61; N, 18.30 .

7-(4-chloro-3-nitrophenyl)-5-isopropyl-N-(4-(3-oxomorpholino)phenyl)-4,7-dihydro$[1,2,4]$ triazolo[1,5-a]pyrimidine-6-carboxamide (4s): White solid; $\mathrm{mp} 266-268{ }^{\circ} \mathrm{C} ; \mathrm{R}_{\mathrm{f}} 0.36$ (4:6 hexane-EtOAc);IR (KBr):3260, 3190, 3072, 2949, 2880, 1750, 1660, 1550, 1470, 1340, 1310, 1280, 1180, 1073, 884, 779, 678, $590 \mathrm{~cm}^{-1}$; MS $(\mathrm{m} / \mathrm{z}): 537\left(\mathrm{M}^{+}\right)$; Anal. Calcd for $\mathrm{C}_{25} \mathrm{H}_{24} \mathrm{ClN}_{7} \mathrm{O}_{5}$ : C, 55.82; H, 4.50; N, 18.23; Found: C, 55.90; H, 4.55; N, 18.35 .

5-isopropyl-N-(4-(3-oxomorpholino)phenyl)-7-(2-(trifluoromethyl)phenyl)-4,7-dihydro$[\mathbf{1 , 2 , 4}]$ triazolo[1,5-a]pyrimidine-6-carboxamide (4t): White solid; mp $234-236{ }^{\circ} \mathrm{C} ; \mathrm{R}_{\mathrm{f}} 0.48$ (4:6 hexane-EtOAc); IR (KBr): 3260, 3188, 3067, 2935, 2875, 1710, 1665, 1583, 1539, 1487 , 1282, 1154, 1093, 1040, 885, 796, 680, $595 \mathrm{~cm}^{-1}$; MS $(\mathrm{m} / \mathrm{z}): 526\left(\mathrm{M}^{+}\right)$; Anal. Calcd for $\mathrm{C}_{26} \mathrm{H}_{25} \mathrm{~F}_{3} \mathrm{~N}_{6} \mathrm{O}_{3}$ : C, 59.31; H, 4.79; N, 15.96; Found: C, 59.39; H, 4.69; N, 16.01 . 
Table 1. Synthesis of substituted Triazolopyrimidines.

\begin{tabular}{|c|c|c|c|c|}
\hline Entry & Code & $\mathbf{R}$ & Yield (\%) & Time (min.) \\
\hline 1 & $4 a$ & $\mathrm{H}$ & 75 & 12 \\
\hline 2 & $4 b$ & $4-\mathrm{Cl}$ & 78 & 13 \\
\hline 3 & $4 c$ & $4-\mathrm{F}$ & 80 & 13 \\
\hline 4 & $4 d$ & $3-\mathrm{Cl}$ & 68 & 14 \\
\hline 5 & $4 \mathrm{e}$ & $2,5-\mathrm{di}-\mathrm{CH}_{3}$ & 80 & 15 \\
\hline 6 & $4 \mathrm{f}$ & $4-\mathrm{CH}_{3}$ & 78 & 15 \\
\hline 7 & $4 \mathrm{~g}$ & 3,4 -di- $\mathrm{OCH}_{3}$ & 69 & 15 \\
\hline 8 & $4 \mathrm{~h}$ & $4-\mathrm{OCH}_{3}$ & 88 & 12 \\
\hline 9 & $4 \mathrm{i}$ & $3-\mathrm{NO}_{2}$ & 84 & 13 \\
\hline 10 & $4 j$ & 2,4-di-Cl & 83 & 16 \\
\hline 11 & $4 \mathrm{k}$ & 3,4-di- $\mathrm{CH}_{3}$ & 73 & 15 \\
\hline 12 & 41 & $2-\mathrm{Cl}$ & 82 & 13 \\
\hline 13 & $4 m$ & $3-\mathrm{CH}_{3}$ & 76 & 14 \\
\hline 14 & $4 n$ & 2,3-di- $\mathrm{CH}_{3}$ & 73 & 14 \\
\hline 15 & 40 & $2-\mathrm{F}$ & 71 & 16 \\
\hline 16 & $4 p$ & $2-\mathrm{NO}_{2}$ & 83 & 18 \\
\hline 17 & $4 q$ & $3-\mathrm{Cl}, 4-\mathrm{F}$ & 85 & 17 \\
\hline 18 & $4 r$ & $4-\mathrm{Cl}, 2-\mathrm{NO}_{2}$ & 72 & 16 \\
\hline 19 & $4 s$ & $4-\mathrm{Cl}, 3-\mathrm{NO}_{2}$ & 70 & 15 \\
\hline 20 & $4 \mathrm{t}$ & $2-\mathrm{CF}_{3}$ & 76 & 15 \\
\hline
\end{tabular}

\section{Biology:}

\section{Antimicrobial Sensitivity Testing Well Diffusion/Agar Cup Method}

In vitro affectivity of antimicrobial agents can be demonstrated by observing their capacity to inhibit bacterial growth on suitable media. The production of a zone depends on two factors namely bacterial growth and concentration of antimicrobial agent. The hole/well punch method was first used by Bennett. This diffusion method has proved more effective 
than many other methods. According to Lt. General Raghunath the well technique is 5-6 times more sensitive than using disk method.

Table 2. Antimicrobial Sensitivity Assay.

\begin{tabular}{|c|c|c|c|c|c|c|c|c|}
\hline \multirow{3}{*}{$\begin{array}{l}\text { Sr. } \\
\text { No. }\end{array}$} & \multirow{3}{*}{ Code no. } & \multicolumn{7}{|c|}{$\operatorname{MIC}(\mu \mathrm{g} / \mathrm{mL})$} \\
\hline & & \multicolumn{4}{|c|}{ antibacterial activity } & \multicolumn{3}{|c|}{ antifungal activity } \\
\hline & & E.coli & P.aeruginosa & S.aureus & S.pyogenus & C.albicans & A.niger & A.clavatus \\
\hline 1 & $4 a$ & 250 & 200 & 250 & 100 & 500 & 1000 & 1000 \\
\hline 2 & $4 \mathrm{~b}$ & 200 & 125 & 100 & 125 & 1000 & $>1000$ & $>1000$ \\
\hline 3 & $4 c$ & 100 & 100 & 125 & 250 & 250 & $>1000$ & $>1000$ \\
\hline 4 & $4 d$ & 125 & 250 & 250 & 100 & 100 & 500 & 500 \\
\hline 5 & $4 e$ & 200 & 250 & 250 & 250 & 1000 & $>1000$ & $>1000$ \\
\hline 6 & $4 \mathrm{f}$ & 62.5 & 200 & 100 & 200 & $>1000$ & 500 & 500 \\
\hline 7 & $4 \mathrm{~g}$ & 100 & 62.5 & 125 & 100 & $>1000$ & 250 & 250 \\
\hline 8 & $4 \mathrm{~h}$ & 250 & 200 & 250 & 250 & 500 & 1000 & 1000 \\
\hline 9 & $4 \mathrm{i}$ & 125 & 200 & 50 & 100 & 250 & $>1000$ & $>1000$ \\
\hline 10 & $4 j$ & 100 & 100 & 100 & 125 & 250 & 1000 & 1000 \\
\hline 11 & $4 \mathrm{k}$ & 50 & 100 & 250 & 250 & 1000 & $>1000$ & $>1000$ \\
\hline 12 & 41 & 100 & 250 & 100 & 200 & 1000 & $>1000$ & $>1000$ \\
\hline 13 & $4 \mathrm{~m}$ & 250 & 500 & 500 & 100 & 1000 & 1000 & 1000 \\
\hline 14 & $4 n$ & 250 & 1000 & 250 & 500 & 1000 & 500 & 500 \\
\hline 15 & 40 & 100 & 250 & 200 & 250 & 500 & $>1000$ & $>1000$ \\
\hline \multicolumn{2}{|c|}{ Gentamycin } & 0.05 & 1 & 0.25 & 0.5 & - & - & - \\
\hline \multicolumn{2}{|c|}{ Ampicilin } & 100 & - & 250 & 100 & - & - & - \\
\hline \multicolumn{2}{|c|}{ Chloramphenicol } & 50 & 50 & 50 & 50 & - & - & - \\
\hline \multicolumn{2}{|c|}{ Ciprofloxacin } & 25 & 25 & 50 & 50 & - & - & - \\
\hline \multicolumn{2}{|c|}{ Norfloxacin } & 10 & 10 & 10 & 10 & - & - & - \\
\hline \multicolumn{2}{|c|}{ Nystatin } & - & - & - & - & 100 & 100 & 100 \\
\hline \multicolumn{2}{|c|}{ Greseofulvin } & - & - & - & - & 500 & 100 & 100 \\
\hline
\end{tabular}




\section{CONCLUSION}

In summary, we have described the synthesis of substituted triazolopyrimidine derivatives in moderate yield. The reaction of various aldehydes with acetoacetamide and 5amino-1,2,4-triazole was afforded the triazolopyrimidine derivatives in moderate to good yield. All the synthesized compounds were evaluated for their antimicrobial activity. The investigation of antibacterial and antifungal screening data revealed that all the tested compounds 4a-t showed moderate to potent activity. The compounds $4 \mathrm{f}, 4 \mathrm{~g}$ and 4 iare found comparatively good active against all the bacterial strains.

\section{Acknowledgements}

Authors are thankful to UGC, New Delhi for the financial support.

\section{References}

[1] Fischer G., Adv. Heterocyclic Chemistry 57 (1993) 81.

[2] Shaban M.A.E., Morgan A.E.A., Adv. Heterocyclic Chemistry 77 (2000) 345.

[3] Shaban, M.A.E., Morgan A.E.A., Adv. Heterocyclic Chemistry 73 (2000) 131.

[4] Shaban, M.A.E.; Morgan A.E.A., Adv. Heterocyclic Chemistry 75 (2000) 243.

[5] Ashour H. M., Shaaban O. G., Rizk O. H., El-Ashmawy I. M., Euro. J. Med. Chem. 62 (2013) 341.

[6] Marwaha A., White J., El Mazoun F., Creason S. A., Kokkond S., Buckner F. S., Rathod P. K., J. Med. Chem. 55 (2012) 7425.

[7] Chen Q., Zhu X. L., Liu, et al., Euro. J. Med. Chem. 43 (2008) 595.

[8] Uryu S., Tokuhiro S., Murasugi T., Brain Research 946 (2002) 298.

[9] Zhang N., Semiramis A. K., Thai N., J. Med. Chem. 50 (2007) 319.

[10] Havlicek L., Fuksova K., Krystof V., Bioorg. Med. Chem. 13 (2005) 5399.

[11] Zhao X., Zhao Y., Guo S., Song H., Wang D., Gong P., Molecules 12 (2007) 1136.

[12] Iwona L., Marzena F., Tadeusz M., Tadeusz S., Julia J., Dalton Transactions 42 (2013) 6219.

[13] Yin L., Shuai Z., Zhi-Jun L., Hai-Liang Z., Euro. J. Med. Chem. 64 (2013) 54.

[14] Ashraf H. F., Abd El-Wahab, Pharmaceuticals 5 (2012) 745.

[15] Abdel-Aziem A., Sayed El-Gendy M., Abdelhamid A. O., Euro. J. Chem. 3 (2012) 455.

[16] Khera M. K., Cliffe I. A., Mathur T., Prakash O., Bioorg \& Med. Chem. Lett. 21 (2011) 2887.

[17] Fraley M. E., Hoffman W. F., Rubino R. S., Bioorg. Med. Chem. Lett. 12 (2002) 2767.

[18] Antonino L., Ilenia A., Chiara P., Annamaria M., Gaetano D., Maria A. A., Euro. J. Med. Chem. 62 (2013) 416.

[19] Fairfield B. J., Andrew C., Allan J., WO2004108136, 2004. 
[20] G. Thirunarayanan, International Letters of Chemistry, Physics and Astronomy 5 (2014) 89-98.

[21] Nirav M. Shah, Hitendra S. Joshi, International Letters of Chemistry, Physics and Astronomy 6 (2014) 56-60.

[22] Piyush B. Vekariya, Jalpa R. Pandya, Vaishali Goswami, Hitendra S. Joshi, International Letters of Chemistry, Physics and Astronomy 7 (2014) 45-52.

[23] Sagar P. Gami, Kalpesh V. Vilapara, Hasmukh R. Khunt, Jayesh S. Babariya, Yogesh T. Naliapara, International Letters of Chemistry, Physics and Astronomy 11(2) (2014) 127-134.

[24] Hasmukh R. Khunt, Piyush P. Pipaliya, Satish M. Ghelani, Jayesh S. Babariya, Yogesh T. Naliapara, International Letters of Chemistry, Physics and Astronomy 12 (2014) 20-25. 